\title{
Developing a paper-based antigen assay to differentiate between coronaviruses and SARS-CoV-2 Spike variants
}

\author{
Delyan R. Hristov ${ }^{\mathrm{a}}$, Hom Rijal ${ }^{\mathrm{b}}$, Jose Gomez-Marquez, ${ }^{\mathrm{c}}$ and Kimberly Hamad-Schifferli ${ }^{\mathrm{a}} \mathrm{d}^{*}$ \\ ${ }^{a}$ Department of Engineering, University of Massachusetts Boston, Boston, MA, USA \\ ${ }^{\mathrm{b}}$ Department of Chemistry, University of Massachusetts Boston, Boston, MA, USA \\ ${ }^{c}$ Little Devices Lab, Massachusetts Institute of Technology, Cambridge, MA USA \\ ${ }^{\mathrm{d} S c h o o l ~ f o r ~ t h e ~ E n v i r o n m e n t, ~ U n i v e r s i t y ~ o f ~ M a s s a c h u s e t t s ~ B o s t o n, ~ B o s t o n ~ M A, ~ U S A ~}$
}

KEYWORDS Dipstick immunoassay, SARS-CoV-2, COVID-19, rapid diagnostic, paper-based test, gold nanoparticles

\begin{abstract}
COVID-19 first appeared in December of 2019 in Wuhan, China. Since then it has become a global pandemic. A robust and scalable diagnostics strategy is crucial for containing and monitoring the pandemic. RT-PCR is a known, reliable method for COVID-19 diagnostics which can differentiate between SARS-CoV-2 and other viruses. However, PCR is location dependent, time consuming and relatively expensive. Thus, there is a need for a more flexible method which may be produced in an offthe-shelf format and distributed more widely. Paper-based immunoassays can fulfill this function. Here we present the first steps towards a paper-based test which can differentiate between different between the Spike protein of various coronaviruses, SARSCoV-1, SARS-CoV-2 and CoV-HKU1 with negligible cross reactivity for HCoV-OC43 and HCoV-229E in a single assay which takes less than 30 minutes. Furthermore, our test can distinguish between fractions of the same Spike protein. This is done by an altered assay design with four test line locations where each antigen builds a unique, identifiable binding pattern. The effect of several factors, such as running media, immunoprobe concentration and antigen interference is considered. We find that running media has a significant effect on the final binding pattern where human saliva provides results while human serum leads to the lowest signal quality.
\end{abstract}

\section{Introduction}

The COVID-19 global pandemic which emerged in Wuhan, China in December of 2019 has infected millions of people and caused or contributed to the death of hundreds of thousands and led to an economic downturn. Diagnosis of COVID19 using diagnostic tools is preferable over clinical symptoms due to the nonspecific nature of COVID-19 symptomology. The majority of patients suffer non-specific, mild symptoms mostly consisting of cough, fever, muscle pain and nausea. ${ }^{1-2}$ Additionally, an estimated $20-80 \%$ of patients are asymptomatic, with varying reports. ${ }^{3-5}$ Traditional laboratory diagnostics such as RT-PCR have high sensitivity and diagnostic accuracy, but are expensive and time-consuming, and may not be locally available. Paper-based immunoassays can compliment PCR and contribute to a better diagnostics strategy. While they commonly suffer from relatively low selectivity and especially sensitivity, compared to PCR, paper-based tests are cheap to produce locally or in a at-scale manufacturing facility, can be made in an off-the-shelf format and stored for prolonged periods of time in mild conditions, e.g. room temperature or $4{ }^{\circ} \mathrm{C} .{ }^{6}$ Furthermore, ther flexible design allows simultaneous detection of multiple targets. ${ }^{7-8}$
Paper-based tests for SARS-CoV-2 detection can be classified as antibody (or serological) tests, i.e. those which measure the host immune response, and antigen tests, i.e. those which bind to viral antigens. ${ }^{9}$ Antigen paper-based immunoassays typically target the Spike (S) or Nucleocapsid (N) proteins. The $\mathrm{S}$ protein decorates the outside of coronaviruses and enables their uptake into cells. ${ }^{10}$ Variances in S protein structure determine the cellular uptake pathway and affinity of the virus for the cell. SARS-CoV-2, similarly to SARS-CoV-1 and HCoV-NL63, targets the ACE2 receptor in the oral and nasal cavities as its primary root of infection. ${ }^{11-12}$ The $\mathrm{S}$ protein has been shown to be a primary driver of viral evolution, where mutations greatly impact virus infection rate and is considered to be the primary difference in reproductive number between clades. $^{13-14}$

A low-cost, rapid, and user-friendly platform which can differentiate is desirable. Distinguishing between respiratory viruses with similar clinical symptoms is especially important not only to provide timely treatment but also better assess patient risk. Here, we present the first step towards developing a paper-based sensor which can differentiate between $\mathrm{S}$ proteins from different coronaviruses as well as Spike protein variants from SARS-CoV-2. The test relies on a sandwich 
immunoassay and antibody cross-reactivity for antigen specific test patterns. We used a set of six commercially available antibodies and seven commercially available $\mathrm{S}$ proteins to establish the viability of the strategy. The test was able to differentiate between all antigens, including SARS-CoV-2 Spike fragments. Test limit of detection (LOD) was in the $0.1 \mathrm{nM}$ range, which may be sufficient for viral detection in more severe cases. We also studied the effect of running medium on test efficacy, bovine serum albumin (BSA) human serum (HS) and saliva.

\section{Results}

\section{Immunoprobe Screening}

We chose spike protein as a target because it protrudes outside the viral particle and is responsible for receptor recognition. It is composed of two parts, S1 and S2, where the receptor binding domain (RBD) is in S1. ${ }^{16} \mathrm{We}$ obtained $\mathrm{S} 1$ proteins for SARS-CoV-2, SARS-CoV-1 (SARS) and non-lethal coronaviruses CoV-HKU1 (HKU1), HCoV-OC43 (OC43) and HCoV-229E (229E) to study the selectivity of our nanoparticle-antibody conjugates (NP-Ab conjugates or immunoprobes). SARS-CoV-2 was termed COVID to avoid confusion (Table 1 and $\mathbf{S 1}$ ).

Table 1. Antigens, antibodies and NP-Ab conjugates used

\begin{tabular}{|l|l|l|l|l|}
\hline \multicolumn{2}{|l|}{ Antigens } & \multicolumn{2}{l|}{ Antibodies } \\
\hline Antigen & Virus & Antibody & NP-Ab & Virus \\
\hline COVID 1 & SARS-CoV-2 & $\alpha \mathrm{C} 1$ & NP- $\alpha \mathrm{C} 1$ & SARS-CoV-2 \\
\hline COVID 2 & SARS-CoV-2 & $\alpha \mathrm{C} 2$ & NP- $\alpha \mathrm{C} 2$ & SARS-CoV-2 \\
\hline COVID 3 & SARS-CoV-2 & $\alpha \mathrm{S} 1$ & NP- $\alpha$ S1 & SARS-CoV-1 \\
\hline SARS & SARS-CoV-1 & $\alpha \mathrm{S} 2$ & NP- $\alpha$ S2 & SARS-CoV-1 \\
\hline $229 E$ & HCoV-229E & $\alpha \mathrm{S} 3$ & NP- $\alpha$ S3 & SARS-CoV-1 \\
\hline OC43 & HCoV-OC43 & $\alpha \mathrm{H}$ & NP- $\alpha \mathrm{H}$ & CoV-HKU1 \\
\hline HKU1 & CoV-HKU1 & & & \\
\hline
\end{tabular}

All antibodies used were commercially available. Six antibodies were evaluated for antigen binding and sandwich immunoassay formation (Table 1 and S2). Two were raised against SARS-CoV-2 ( $\alpha \mathrm{C} 1-2)$, three for SARS ( $\alpha \mathrm{S} 1-3)$, and one for CoV-HKU1 $(\alpha H)$. Gold NP-Ab conjugates were synthesized using literature methods. ${ }^{17-18}$ Briefly, each antibody was conjugated to the NPs by physisorption, and then thiolated PEG was added after conjugation to backfill open areas on the NP surface. Dynamic light scattering (DLS) and UV-Vis spectroscopy were used to determine colloidal stability and size dispersion in PBS. DLS was used to measure particle size in BSA, saliva and HS. DLS of NPs showed hydrodynamic diameters $\left(D_{H}\right)$ of $\sim 65 \mathrm{~nm}$ which increased $\sim 85 \mathrm{~nm}$ after antibody conjugation, supporting conjugation (SI Figures S1, S2). The size further increased to $120-200 \mathrm{~nm}$ in media, possibly due to protein adsorption to the NP surface.

Antibody target and off-target binding were studied using antigen and sandwich dipstick assays. In the former case the antigens were immobilized on nitrocellulose following which the prepped strips were immersed in an immunoprobe dispersion (Figure 1a). In the latter case sandwich immunoassay formation, that is binding between the paper printed antibody, antigen and immunoprobe, was tested (Figure 1c). $\alpha$-rabbit IgG Fc $(\alpha-\mathrm{Fc})$, was immobilized at the control area during both tests. A signal at the test line certified fluid flowed through the paper strip. Localized signal emerges on the strip due to immunoprobe accumulation (Figure 1a and c). NP$\alpha \mathrm{S} 2$ and NP- $\alpha \mathrm{S} 3$ NPs were eliminated due to poor performance in initial tests (SI Figure S3).

After running, strips were left to dry overnight at room temperature after which they were scanned and analyzed via ImageJ. ${ }^{19}$ The resulting grayscale values were normalized to $100 \%$ and plotted as a heatmap for clarity (Figure 1b), where white represents low intensity $(<20 \%$ of maximum value) and green high. Averaged grayscale values are provided in the Supporting Information.

In antigen binding tests, NP- $\alpha \mathrm{C} 1$ and NP- $\alpha \mathrm{C} 2$ bound to both COVID and SARS antigens, but not other coronavirus antigens. NP- $\alpha \mathrm{S} 1$ bound to its target antigen, SARS, and exhibited some cross reactivity for COVID 1 and OC43. Immunoprobe binding in saliva resulted in similar signal intensities, but were notably lower in human serum (HS) (Figure 1b, S4 and S5), which could be attributed to HS screening of the immunoprobe function. ${ }^{9}$

We evaluated the ability of the immunoprobes to form an immunological sandwich in a dipstick assay (Figure 1c). ${ }^{20}$ Sandwich formation relies on two reactions, immunoprobeantigen and printed antibody-antigen binding. The choice of both antibodies is primary during test design, where antibody pairs (on the immunoprobe / printed on the paper) with varying affinity and selectivity for a target can be used to detect a wider variety of antigens and develop a binding pattern. ${ }^{21}$ In this initial study we screened the pairing of an antibody with itself, i.e., NP- $\alpha \mathrm{S} 1$ run with immobilized $\alpha \mathrm{S} 1$ (NP- $\alpha \mathrm{S} 1 / \alpha \mathrm{S} 1)$, $\mathrm{NP}-\alpha \mathrm{C} 1$ with $\alpha \mathrm{C} 1(\mathrm{NP}-\alpha \mathrm{C} 1 / \alpha \mathrm{C} 1)$, NP- $\alpha \mathrm{C} 2$ with $\alpha \mathrm{C} 1$ (NP$\alpha \mathrm{C} 2 / \alpha \mathrm{C} 1)$, and NP- $\alpha \mathrm{H}$ with $\alpha \mathrm{H}(\mathrm{NP}-\alpha \mathrm{H} / \alpha \mathrm{H}), \alpha \mathrm{C} 2$, a monoclonal antibody, did not form a sandwich with itself.

$\mathrm{NP}-\alpha \mathrm{C} 1 / \alpha \mathrm{C} 1$ and $\mathrm{NP}-\alpha \mathrm{C} 2 / \alpha \mathrm{C} 1$ were able to successfully form sandwich immunoassays with both COVID and SARS antigens, as indicated by the color resulting at the test line (Figure 1d, S6 and S7). NP- $\alpha \mathrm{S} 1 / \alpha \mathrm{S} 1$ was able to detect the SARS antigen, but not COVID antigens. The observed difference in behavior is potentially due to protein folding. NP$\alpha \mathrm{C} 1 / \alpha \mathrm{C} 1$ and $\mathrm{NP}-\alpha \mathrm{C} 2 / \alpha \mathrm{C} 1$ exhibited no cross reactivity for $229 \mathrm{E}$, OC43, and HKU1, and $\mathrm{NP}-\alpha \mathrm{H} / \alpha \mathrm{H}$ exhibited signal only with HKU1.

Pairs were also tested in saliva and HS (Figure 1d), and the behavior was largely similar to BSA except for NP- $\alpha \mathrm{H} / \alpha \mathrm{H}$ exhibiting some cross reactivity towards OC43 in saliva. Saliva resulted in similar intensities to BSA, but intensities were significantly lower in HS, highlighting the importance of the biological media. Generally, direct antigen binding had a higher signal and cross reactivity than sandwich formation (Figure 1b and d). 

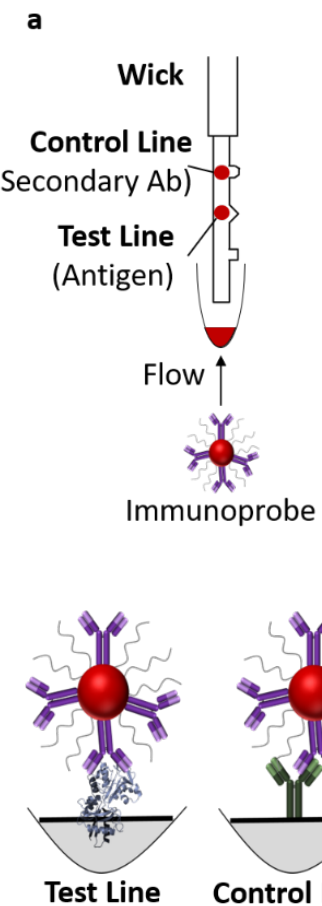

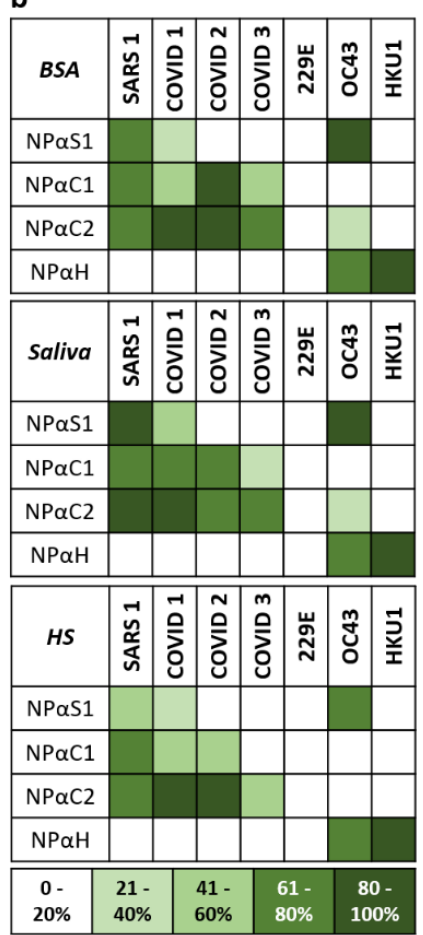

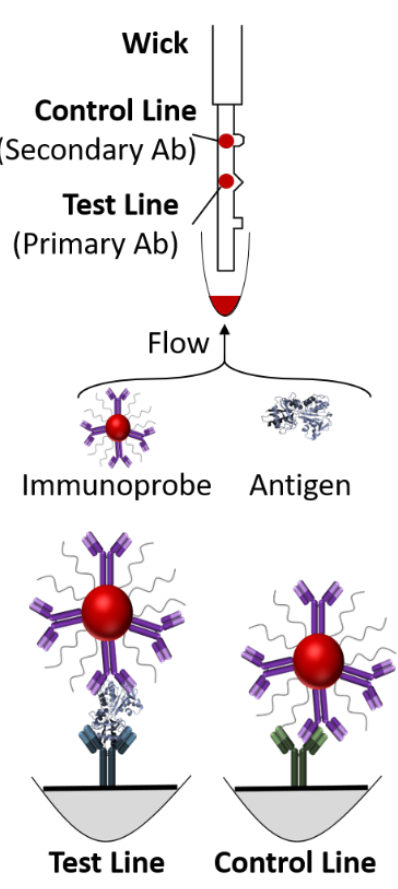

d

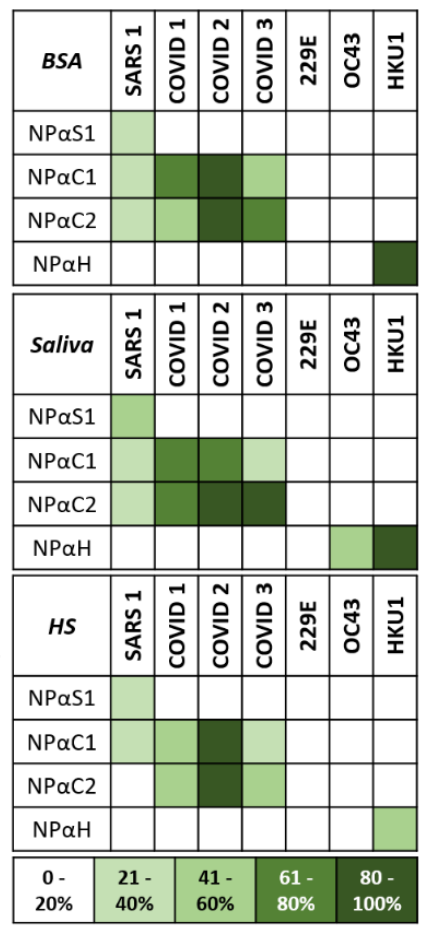

Figure 1. Antibody screening. a) Experimental setup for direct antigen binding tests and b) average test area intensities run in triplicate in BSA, saliva, and HS; c) Experimental setup for sandwich immunoassays, d) average test area intensities in BSA, saliva, and HS. Intensities were of all strips were normalized. Averaged results are from at least three independent batches. Related grayscale values and standard deviations are shown in SI Figure S5 and S7.

Cross reactivity was more commonly observed in saliva compared to the other media. This could be attributed to slower flow possibly due to surface tension, ${ }^{22}$ which results in longer residence time of the immunoprobe-antigen complex near the test areas, and increases binding probability. A compounding factor could be saliva composition, which has different surfactants or macromolecules and a lower $\mathrm{pH}(6-7)^{23-24}$ compared to BSA and HS (7.4). We expect another large contributor to be the relatively low protein concentration of saliva, which is $>99 \%$ water (wt $/ \mathrm{wt}$ ) and $\sim 1 \mathrm{mg} / \mathrm{mL}$ protein $^{22}$ compared to BSA $(30 \mathrm{mg} / \mathrm{mL})$ and $\mathrm{HS}(60-80 \mathrm{mg} / \mathrm{mL})$. Test backgrounds in all three media were similar, suggesting low non- specific binding of the immunoprobes to the paper.

Antigen cross reactivity could be attributed to proximity in phylogeny. Cross reactivity between SARS and SARS-CoV-2 antibodies and antigens was expected due to $\sim 82 \%$ sequence similarities, ${ }^{13,} 25-26$ while the proximity between the RBD regions is $\sim 73 \%{ }^{13} \mathrm{NP}-\alpha \mathrm{H}$ bound to its target antigen HKU1, as well as OC43 presumably due to proximity in phylogeny. ${ }^{10}$ Antigen sequences were compared between each other and the Wuhan reference strain structure (accession number YP_009724390.1) using MUSCLE (SI Figure S8a). Structural differences in MUSCLE can arise from both sequence length and content therefore it is expected that some structural dissimilarities arise our antigens being recombinant fractions of the $\mathrm{S}$ protein. HKU1 was most dissimilar shoring the most structural similarity with the Wuhan reference strain $(20 \%)$ and the least with COVID $1(10 \%)$. SARS had a $\sim 50 \%$ structural similarity with COVID 1 and 2 and $\sim 28 \%$ with COVID 3 and the Wuhan reference train. Similarity between the COVID antigens and compared with the Wuhan strain were $30-50 \%$. Comparison of the RBD of all COVID antigens revealed a
$100 \%$ structural similarity (SI Figure S8b). COVID 1 had a 98\% similarity with the Wuhan strain (SI Figure S8c).

Quantifying sandwich immunoassay performance in different media

Performance of the sandwich immunoassays was investigated by quantifying their limit of detection (LOD) and effective dissociation constant $\left(K_{D}^{E f f}\right)$ for their target antigens. NP$\alpha \mathrm{C} 1 / \alpha \mathrm{C} 1, \mathrm{NP}-\alpha \mathrm{C} 2 / \alpha \mathrm{C} 1$, and $\mathrm{NP}-\alpha \mathrm{H} / \alpha \mathrm{H}$, were titrated with COVID 1 and HKU1 antigens in BSA, HS and saliva. Test line intensities were measured and fit with a modified Langmuir equation. ${ }^{17-18,27} K_{D}{ }^{E f f}$ was obtained from the fitting. LOD was calculated separately, defined as the background signal + $3 \mathrm{x}$ the standard deviation of the background (SI, Calculations section). LODs ranged from 0.1 to $0.17 \mathrm{nM}$ while $K_{D}^{E f f}$ values were in the $10^{-10} \mathrm{M}$ range (Figure 2a-c, Table 1 and S3).

Changing the running media impacted performance. Compared to BSA, antibody pairs generally performed similarly or better in saliva and worse in HS (Figure 2a-c, Table 1). The LOD of NP- $\alpha \mathrm{C} 1 / \alpha \mathrm{C} 1$ was lowest in BSA and $2 \mathrm{X}$ higher in saliva and $7 \mathrm{x}$ in HS (Figure 2a and Table 1). Titration curves of NP- $\alpha \mathrm{C} 2 / \alpha \mathrm{C} 1$ were lowest in BSA, followed by saliva (3x), and significantly higher in HS (9x) (Figure 2b).

Between the two SARS-CoV-2 pairs, NP- $\alpha \mathrm{C} 1 / \alpha \mathrm{C} 1$ exhibited comparable behavior, but marginally outperformed NP$\alpha \mathrm{C} 2 / \alpha \mathrm{C} 1$ in all media by $\sim 2 \mathrm{X}$. This is consistent with sandwich results where NP- $\alpha \mathrm{C} 1 / \alpha \mathrm{C} 1$ which had a higher intensity with COVID 1 than NP- $\alpha \mathrm{C} 2 / \alpha \mathrm{C} 1$. NP $-\alpha \mathrm{H} / \alpha \mathrm{H}$ behavior changed the most with running media, where LOD and $K_{D}{ }^{E f f}$ values varied by two orders of magnitude between saliva and HS. LODs were $0.07 \mathrm{nM}$ in saliva and $0.54 \mathrm{nM}$ in HS. $K_{D}{ }^{E f f}$ values were $0.06 \mathrm{nM}$ in saliva and $2.78 \mathrm{nM}$ in HS (Table 1, Figure 2c, d). 

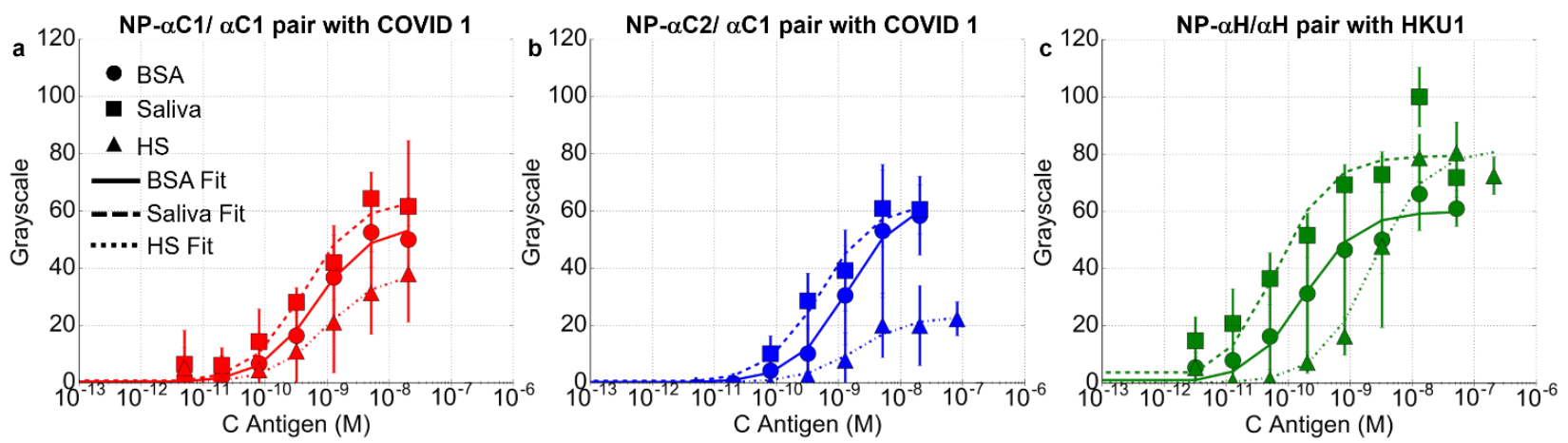

Figure 2. Immunoprobe performance. Titration curves of selected pairs. a) NP- $\alpha \mathrm{C} 1 / \alpha \mathrm{C} 1$ run with COVID $1, b) \mathrm{NP}-\alpha \mathrm{C} 2 / \alpha \mathrm{C} 1$ run with COVID 1, c) NP- $\alpha \mathrm{H} 1 / \alpha \mathrm{H} 1$ run with HKU1.

\section{Control of antigen detection through cross reactivity}

We then investigated how the test could be used a multiplexed test to differentiate between SARS-CoV-2 antigens with similar structure and other coronavirus species, building on the ability to strategically use cross-reactive antibodies to differentiate between different antigens. ${ }^{21} \mathrm{We}$ first studied the relevance of both binding events on sandwich formation and antigen detection. The signal of combinations of all three antigens (SARS, COVID 1 and HKU1), immunoprobes (NP- $\alpha \mathrm{C} 1$, $\mathrm{NP}-\alpha \mathrm{C} 2, \mathrm{NP}-\alpha \mathrm{H})$ and printed antibodies $(\alpha \mathrm{S} 1, \alpha \mathrm{C} 1, \alpha \mathrm{H})$ was measured (total of 27 combinations, Figure S9a). Experiments were repeated in triplicate in BSA at antigen concentrations of $1 \times 10^{-3} \mathrm{mg} / \mathrm{mL}$.

SARS was detectable only with the NP- $\alpha \mathrm{C} 1 / \alpha \mathrm{S} 1$ and NP$\alpha \mathrm{C} 2 / \alpha \mathrm{S} 1$ pairs. COVID 1 was detectable only with the NP$\alpha \mathrm{C} 1 / \alpha \mathrm{C} 1$ and $\mathrm{NP}-\alpha \mathrm{C} 2 / \alpha \mathrm{C} 1$ pairs, and HKU1 was detectable only with the NP- $\alpha \mathrm{H} / \alpha \mathrm{H}$ pair (SI Figure S9b and c). This further confirmed HKU1 was not cross reactive with the other coronavirus antibodies.

These results reaffirm the efficacy of the cross reactivity strategy, where only SARS was detected at immobilized $\alpha \mathrm{S} 1$ and only COVID 1 at immobilized $\alpha \mathrm{C} 1$. We attribute this to the lower affinity of cross reactive interactions, which was confirmed by off target antigen titrations (SI Figure S10). The $\mathrm{NP}-\alpha \mathrm{C} 1 / \alpha \mathrm{C} 1$ and $\mathrm{NP}-\alpha \mathrm{S} 1 / \alpha \mathrm{C} 1$ antibody couples were titrated with their "off target" antigens, being SARS and COVID 1, respectively (SI Figure S10 and Table S4 and S5). Both showed reduced signal in all media compared to their "on target" immunoprobe performance. There was no significant difference in LOD and $K_{D}{ }^{E f f}$ for NP- $\alpha \mathrm{C} 1 / \alpha \mathrm{C} 1$ compared to its "on-target" COVID 1 titration (Table S4 and S5).

Our results suggest that detection of SARS and COVID 1 is more dependent on the printed antibody rather than the one conjugated to the AuNP. On average, there was an order of magnitude drop in "off target" interactions, compared to "on target ones", e.g. NP- $\alpha \mathrm{C} 1 / \alpha \mathrm{S} 1$ to $\mathrm{NP}-\alpha \mathrm{C} 1 / \alpha \mathrm{C} 1$ with SARS (SI Figure S9b-c). Overall these results suggest that cross reactivity can be used for generating patterns.

\section{Multiplexed test}

We designed multiplexed assay to differentiate between spike antigens of SARS, COVID 1, 2 and 3 and simultaneously detect spike from a non-lethal coronavirus, HKU1. The strip consisted of a control and four test areas at differently shaped locations (Figure 3a). Choice of location shape was to differ- entiate locations easier. Test area geometry did not have a measurable impact on signal quality. The antibodies immobilized at the test locations were (bottom to top) $\alpha \mathrm{S} 1$ (square), $\alpha \mathrm{C} 2$ (triangle), $\alpha \mathrm{C} 1$ (octagon) and $\alpha \mathrm{H}$ (oblong). A 1:1 volumetric mixture of NP- $\alpha \mathrm{C} 1$ and $\mathrm{NP}-\alpha \mathrm{H}$ was used to detect all antigens.

Table 2. LOD and $K_{D}{ }^{\text {Eff }}$ values of pairs for target antigens in BSA, saliva, and HS.

\begin{tabular}{|c|c|c|c|c|c|c|}
\hline & $\begin{array}{l}\text { BSA } \\
\text { LOD } \\
(\mathrm{nM})\end{array}$ & $\begin{array}{l}\mathrm{K}_{\mathrm{D}}^{\mathrm{Eff}} \\
(\mathrm{nM})\end{array}$ & $\begin{array}{l}\text { Saliva } \\
\text { LOD } \\
(\mathrm{nM})\end{array}$ & $\begin{array}{l}\mathrm{K}_{\mathrm{D}}{ }^{\mathrm{Eff}} \\
(\mathrm{nM})\end{array}$ & $\begin{array}{l}\text { HS } \\
\text { LOD } \\
(\mathrm{nM})\end{array}$ & $\begin{array}{l}\mathrm{K}_{\mathrm{D}}{ }^{\mathrm{Eff}} \\
(\mathrm{nM})\end{array}$ \\
\hline $\begin{array}{l}\text { NP- } \\
\alpha \mathrm{C} 1 / \\
\alpha \mathrm{C} 1 \\
\text { with } \\
\text { COVID } \\
1\end{array}$ & 0.08 & 0.41 & 0.24 & 0.26 & 0.59 & 0.6 \\
\hline $\begin{array}{l}\text { NP- } \\
\alpha \mathrm{C} 2 / \alpha \mathrm{C} 1 \\
\text { with } \\
\text { COVID } \\
1\end{array}$ & 0.17 & 0.88 & 0.56 & 0.32 & 1.56 & 1.28 \\
\hline $\begin{array}{l}\text { NP- } \\
\alpha \mathrm{H} / \alpha \mathrm{H} \\
\text { with } \\
\text { HKU1 }\end{array}$ & 0.03 & 0.18 & 0.07 & 0.06 & 0.54 & 2.78 \\
\hline
\end{tabular}

Running the assay with the different antigens produced characteristic binding patterns at the four test areas. SARS and COVID antigens were detectable on all locations except the $\alpha \mathrm{H}$ area (oblong) in saliva (Figure 3b). SARS resulted in signal mostly at the $\alpha \mathrm{S} 1$ location, with lower signals at $\alpha \mathrm{C} 1$ and $\alpha \mathrm{C} 2$. COVID 1 exhibited the highest signal at $\alpha \mathrm{C} 2$ and lower signal with $\alpha \mathrm{C} 1$ and $\alpha \mathrm{S} 1$ (Figure S3b). COVID 2 was only detectable at $\alpha \mathrm{C}$ areas with comparable signal intensity at both locations (Figure 3b). As expected from its structure, COVID 3 produced signal mostly at the $\alpha \mathrm{C} 2$ area. 229E and OC43 antigens in saliva yielded no significant signal, and HKU1 was observable only at the $\alpha \mathrm{H}$ test area. 


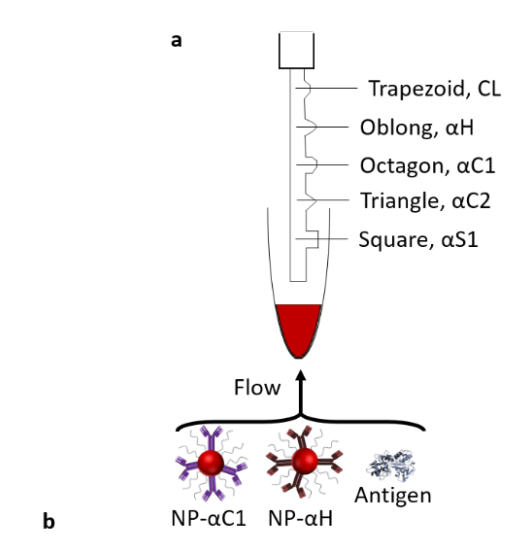

\begin{tabular}{|c|c|c|c|c|c|c|c||c|c|c|c|}
\hline c SARS 1 & BSA & HS & Sal. & COVID 1 & BSA & HS & Sal. & COVID 2 & BSA & HS & Sal. \\
\hline$\alpha H$ & & & & $\alpha H$ & & & & $\alpha H$ & & & \\
\hline$\alpha C 1$ & & & & $\alpha C 1$ & & & & $\alpha C 1$ & & & \\
\hline$\alpha C 2$ & & & & $\alpha C 2$ & & & & $\alpha C 2$ & & & \\
\hline$\alpha S 1$ & & & & $\alpha S 1$ & & & & $\alpha S 1$ & & & \\
\hline
\end{tabular}

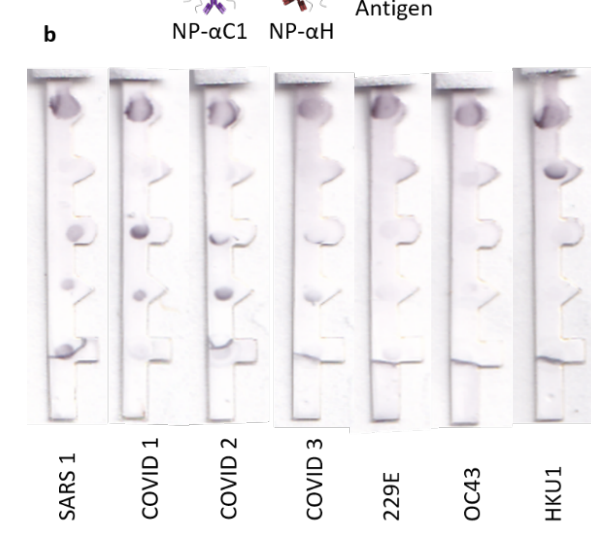

\begin{tabular}{|c|c|c|c|c|c|c|c||c|c|c|c|}
\hline COVID 3 & BSA & HS & Sal. & 229E & BSA & HS & Sal. & OC43 & BSA & HS & Sal. \\
\hline$\alpha H$ & & & & $\alpha H$ & & & & $\alpha H$ & & & \\
\hline$\alpha C 1$ & & & & $\alpha C 1$ & & & & $\alpha C 1$ & & & \\
\hline$\alpha C 2$ & & & & $\alpha C 2$ & & & & $\alpha C 2$ & & & \\
\hline$\alpha S 1$ & & & & $\alpha S 1$ & & & & $\alpha S 1$ & & & \\
\hline
\end{tabular}

\begin{tabular}{|c|l|l|l|}
\hline HKU1 & BSA & HS & Sal. \\
\hline$\alpha H$ & & & \\
\hline$\alpha C 1$ & & & \\
\hline$\alpha C 2$ & & & \\
\hline$\alpha S 1$ & & & \\
\hline
\end{tabular}

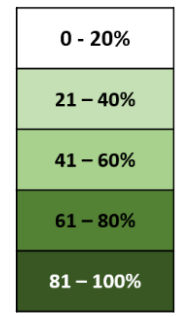

Figure 3. Altered strip design and antigen binding patterns. a) schematic of experimental procedure, b) example strips run in saliva, c) average test area intensities run in triplicate in BSA, saliva, and HS. Intensities of all strips were normalized. Grayscale values for test shown in SI Figure S11.

Again, signals were similar in BSA and saliva but were either reduced in intensity or lost in HS. For example, SARS could not be detected in HS, but displaye a characteristic pattern in BSA and saliva (Figure 3c, top left). HKU1 signal was strongest in BSA and weakest in HS, which was consistent with single strip results (Figures 1d, 2c). Cross reactivity was observed more often in saliva, where SARS was detectable at the $\alpha \mathrm{C} 2$ location, COVID 2 was detectable at the $\alpha \mathrm{S} 1$ location and OC43 was detectable at the $\alpha \mathrm{H}$ location.

\section{Interference between multiple antigens in multiplexed test}

We ran antigen mixtures in our test to investigate binding interference (Figure 4a). When a mixture of SARS + HKU1 was run, intensity appeared at all four test areas, with higher intensity at $\alpha \mathrm{H}$ and $\alpha \mathrm{S} 1$. This pattern resembled the additive intensity pattern of SARS alone plus HKU1 alone (Figure $4 \mathbf{b}$ and SI Figure S13e, i, j). This suggested that the antigens did not compete with each other for forming immunoassay pairs.

Results suggest that the binding pattern in the presence of multiple antigens is similar to mathematically adding the two independent patterns if the two antigens did not bind to the same immunoprobe, e.g. SARS 1 and HKU1 (Figure 4b, S13 and S14).

However, for antigens which bind to the same immunoprobe, the pattern depended on the affinity of the interaction. When a mixture of SARS1 + COVID 1 + HKU1 was run, intensity appeared at all four test areas, showing that the test was able to simultaneously detect SARS, COVID1 and HKU1. However, the intensity pattern did not resemble what would be anticipated from simply adding the intensities of the individually run antigens (Figure 4c). Since SARS and COVID 1 interact with $\mathrm{NP}-\alpha \mathrm{C} 1$ with a similar dissociation constant $\left(K_{D}^{E f f}(\mathrm{SARS})=0.86 \mathrm{nM}, K_{D}^{E f f}(\right.$ COVID 1$)=0.42$ $\mathrm{nM})$ the pattern of the mixture depended on the relative con- centration of the two antigens. In this case, the antigen with the higher concentration would have a more pronounced pattern (SI Figure S12 and S13a, b, f and S14a, b, f). Similar effects were observed for a SARS, COVID 1 and 2 mixture $\left(K_{D}{ }^{\text {Eff }}(\right.$ COVID 2$)=0.10 \mathrm{nM}$, SI Figure S12).

Some challenges arose with the multiplexed strip. Background gradients were more common in longer strips. To accommodate for this, we limited the strip to 5 test locations and modified the image analysis. We immobilized antibodies with higher affinity further up the strip so they would not deplete the immunoprobe/antigen complexes before they encountered lower affinity immobilized antibodies. An optimal immunoprobe or immunoprobe mixture concentration was determined to be $12-17 \%$ of the total sample volume (SI Figure S15).

By understanding and controlling these principles, it was possible to simultaneously detect and differentiate all three coronavirus antigens (Figure 4c, SI Figure S13 and S14).

\section{Discussion}

The paper-based immunoassay investigated here was able to differentiate spike antigens from different coronaviruses by building a binding pattern through the number, arrangement and specificity of printed antibodies. Differentiation between SARS-CoV-1, SARS-CoV-2 and HKU1 S1 proteins was simpler based on their patterns where SARS bound mostly to the $\mathrm{NP}-\alpha \mathrm{C} 1 / \alpha \mathrm{S} 1$ pair while HKU1 bound exclusively to NP$\alpha \mathrm{H} / \alpha \mathrm{H}$. Negligible cross reactivity with $229 \mathrm{E}$ and OC43 was observed. 

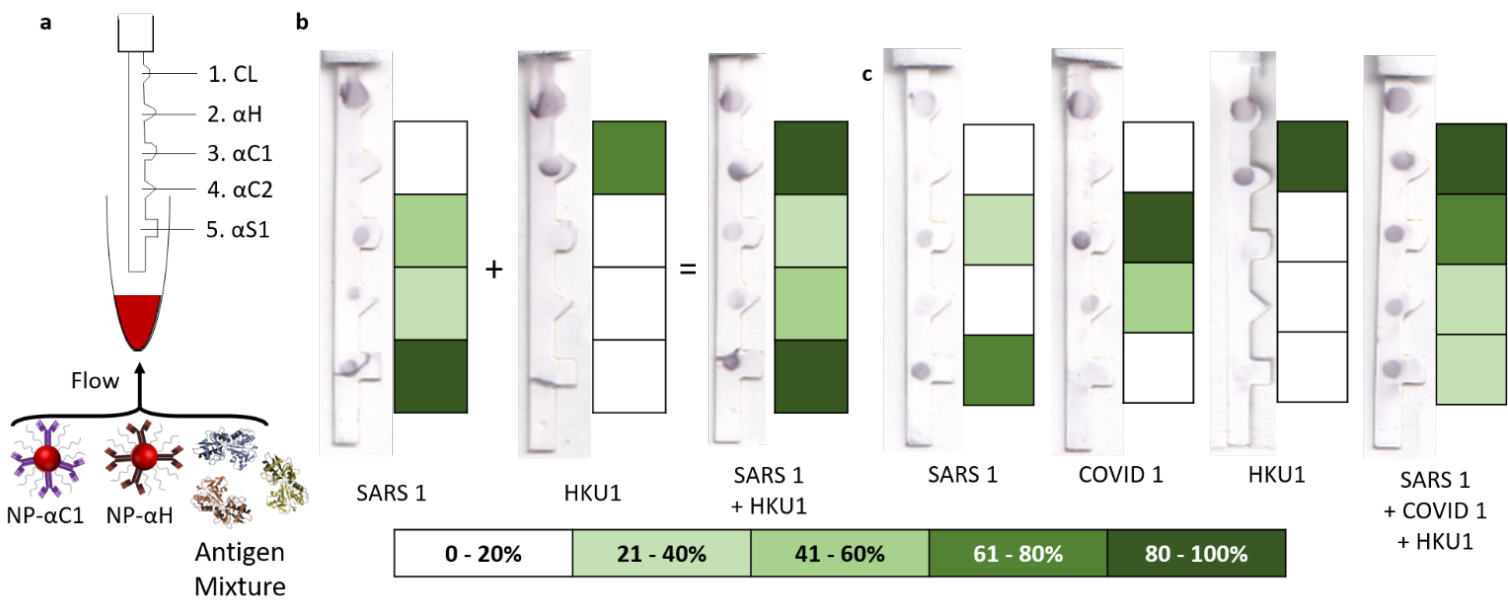

Figure 4. Running antigen mixture in altered strip design. a) Schematic of experimental procedure, b) example strips, patterns and from a test run with SARS $1+$ HKU1 and c) SARS1 + COVID $1+$ HKU1.

The ability to differentiate between SARS-CoV-2 antigens is especially interesting and could yield new information for patient samples. We applied our test to three recombinant $\mathrm{S} 1$ fragments, COVID 1 with a length of 681 amino acids (aa), COVID 2 with 461 aa and COVID 3 with 229. Considering the high similarity in sequence (SI Figure S8) the difference in binding pattern can be attributed to their size. Thus, the use of binding patterns may be able to differentiate between different Spike products in patient samples. This may help address some of the questions raised about patient viral shedding over time. ${ }^{28}$ Differentiation between Spike protein variants or fractions may be improved by increasing the specificity and number of antibodies printed on the nitrocellulose. It is unlikely that the test design would be able to differentiate between Spike proteins from different clades due to high sequence similarity $(>99 \%)$.

The ability to detect and differentiate between antigen from other coronaviruses and antigen mixtures should not be overlooked. Co-circulation of non-lethal coronaviruses, other respiratory viruses and SARS-CoV-2 has been reported. ${ }^{29-31} \mathrm{Co}-$ infections between SARS-CoV-2 with other respiratory viruses are rare, reported in $3-20 \%$ of cases, including co-infections with non-lethal coronaviruses in about $0.1-5 \%$ of cases. ${ }^{32-33}$ However, rare a recent study in the UK suggests that coinfection of SARS-CoV-2 with infuenza has been shown to increase risk for poor patient outcomes. ${ }^{29}$ Co-infection may also help explain reported abnormal viral shedding patterns and differences in patient outcomes, so a test that can simultaneously differentiate between common respiratory viruses would help address these questions.

The running media impacted antigen binding, where strips run in BSA and saliva had comparable results with a $15 \%$ variation in signal, lower than test-to-test variability. Running assays in HS resulted in a reduction or complete loss of signal, which could be attributed to protein screening effects. ${ }^{17,34}$ LODs for COVID antigens in BSA and saliva were in the $\sim \mathrm{ng} / \mathrm{mL}$ range $(0.03$ to $0.56 \mathrm{nM})$. In comparison, others have reported similar performance with a LOD of $0.62 \mathrm{ng} / \mathrm{ml}$ $(0.0125 \mathrm{nM})$ for half strips for SARS-CoV-2 nucleocapsid $(\mathrm{N})$ proteins. ${ }^{20,35}$ We caveat that the LOD measurement was based on purified antigens in solution and not the full virus capsid in biological fluids, and matrix effects in patient samples or differences in spike secondary structure could influence performance. We estimate that the average number of Spike proteins in sputum swabs may be in the $\mathrm{pM}$ range for most cases and
$\mathrm{nM}$ range in more severe cases. This estimate is based on the average copies of RNA per $\mathrm{mL}$ of saliva ${ }^{36}$ and the average number of spike proteins on a single virus. ${ }^{37} \mathrm{We}$ assume that every RNA copy corresponds to a virus, that each virus has 100 Spikes on its surface and there are no free Spike proteins. This is a rough estimate due to the assumptions made (SI Calculations section). While the concentration of spike protein in patient samples over time has still not been definitively quantified, it is anticipated that the LOD here may be too high for visual readouts for most patient samples. ${ }^{37-40}$

The intensity in a paper-based sandwich immunoassay depends on multiple factors, such as antibody affinity, immunoprobe concentration, antibody coverage on the NP, its size, concentration of the immobilized capture antibody, and several others. ${ }^{9}$ Varying these parameters can improve signal intensity without external enhancement approaches. Commercial diagnostics have solved this with dedicated readers for colorimetric or fluorescent readouts (Sofia, Quidel Corp.). Surface enhanced Raman spectroscopy (SERS) nanotags, ${ }^{41-42}$ isotachaphoresis, ${ }^{43}$ and photothermal heating ${ }^{44}$ have all been used successfully to increase signal, sometimes as high as 100 -fold. Simply running the flow back and forth over multiple passes can increase signal 5-fold, and does not require additional readout instrumentation. ${ }^{7}$ Additionally, concentrating the antigen by sample preparation techniques or paperfluidic design could increase signal. Ultimately, this sensitivity gap could be solved by a combination of techniques. ${ }^{45}$

Test variability was estimated through the standard deviation and relative standard deviation (RSD) from all tests (SI Figure S16), and was estimated to be $36 \%$, which did not change significantly between media or antigen mixtures. Typically, lower signals and weaker antigen-antibody interactions resulted in higher RSDs.

Results presented here can be used towards the development of COVID-19 paper-based dipstick and lateral flow assays (LFAs), a rapid diagnostic format that has aided in disease management, quarantine, and surveillance. The low production cost and relative ease of use of paper rapid diagnostics makes them suitable for both local and large scale production, making it a potentially powerful off-the-shelf complement to RT-PCR, which could help elevate strain on local diagnostic facilities to meet the massive demand for tests. The selfcontained nature of paper-based tests makes them attractive for remote or mobile locations. 


\section{ASSOCIATED CONTENT}

Supporting Information contains secondary figures and tables providing context for the text, calculations used and methods section. This material is available free of charge via the Internet at http://pubs.acs.org.

\section{AUTHOR INFORMATION}

\section{Corresponding Author}

*kim.hamad@umb.edu

\section{Author Contributions}

DRH designed and carried out the studies, and wrote and edited the manuscript, HR and JGM wrote and edited the manuscript, KHS designed the studies, wrote and edited the manuscript.

\section{Funding Sources}

This work was supported by UMass Boston.

\section{ACKNOWLEDGMENT}

We thank UMass Boston for support during the pandemic.

\section{ABBREVIATIONS}

BSA, bovine serum albumin; DLS, dynamic light scattering; HS, human serum; LOD, limit of detection; NP, nanoparticle; RSD, relative standard deviation.

\section{REFERENCES}

1. $\mathrm{Fu}, \mathrm{L} . ;$ Wang, B.; Yuan, T.; Chen, X.; Ao, Y.; Fitzpatrick, T.; Li, P.; Zhou, Y.; Lin, Y.-f.; Duan, Q.; Luo, G.; Fan, S.; Lu, Y.; Feng, A.; Zhan, Y.; Liang, B.; Cai, W.; Zhang, L.; Du, X.; Li, L.; Shu, Y.; Zou, H., Clinical characteristics of coronavirus disease 2019 (COVID-19) in China: A systematic review and meta-analysis. Journal of Infection 2020, 80 (6), 656-665.

2. Docherty, A. B.; Harrison, E. M.; Green, C. A.; Hardwick, H. E.; Pius, R.; Norman, L.; Holden, K. A.; Read, J. M.; Dondelinger, F.; Carson, G.; Merson, L.; Lee, J.; Plotkin, D.; Sigfrid, L.; Halpin, S.; Jackson, C.; Gamble, C.; Horby, P. W.; Nguyen-Van-Tam, J. S.; Ho, A.; Russell, C. D.; Dunning, J.; Openshaw, P. J. M.; Baillie, J. K.; Semple, M. G., Features of 20133 UK patients in hospital with covid19 using the ISARIC WHO Clinical Characterisation Protocol: prospective observational cohort study. BMJ 2020, 369, m1985.

3. Buitrago-Garcia, D.; Egli-Gany, D.; Counotte, M. J.; Hossmann, S.; Imeri, H.; Ipekci, A. M.; Salanti, G.; Low, N., Occurrence and transmission potential of asymptomatic and presymptomatic SARS-CoV-2 infections: A living systematic review and meta-analysis. PLOS Medicine 2020, 17 (9), e1003346.

4. Ing, A. J.; Cocks, C.; Green, J. P., COVID-19: in the footsteps of Ernest Shackleton. Thorax 2020, 75 (8), 693.

5. Petersen, I.; Phillips, A., Three Quarters of People with SARS-CoV-2 Infection are Asymptomatic: Analysis of English Household Survey Data. Clinical Epidemiology 2020, 12 (12), 10391043.

6. Wang, J.; Yiu, B.; Obermeyer, J.; Filipe, C. D. M.; Brennan, J. D.; Pelton, R., Effects of Temperature and Relative Humidity on the Stability of Paper-Immobilized Antibodies. Biomacromolecules 2012, 13 (2), 559-564.

7. Phillips, E.; Young, A. K.; Albarran, N.; Butler, J.; Lujan, K.; Hamad-Schifferli, K.; Gomez-Marquez, J., Ampli: A Construction Set for Paperfluidic Systems. Advanced Healthcare Materials 2018, 1800104.

8. Sanchez-Purra, M.; Roig-Solvas, B.; Rodríguez-Quijada, C.; Leonardo, B.; Hamad-Schifferli, K., Design of Nanotags for
Multiplexed Surface Enhanced Raman Spectroscopy Assays. ACS Omega 2018, 3 (9), 10733-10742.

9. Hristov, D. R.; Rodríguez-Quijada, C.; Gomez-Marquez, J.; Hamad-Schifferli, K., Designing Paper-Based Immunoassays for Biomedical Applications. Sensors 2019, 19 (3), 554.

10. Chen, B.; Tian, E.-K.; He, B.; Tian, L.; Han, R.; Wang, S.; Xiang, Q.; Zhang, S.; El Arnaout, T.; Cheng, W., Overview of lethal human coronaviruses. Signal Transduction and Targeted Therapy 2020, 5 (1), 89.

11. Radzikowska, U.; Ding, M.; Tan, G.; Zhakparov, D.; Peng, Y.; Wawrzyniak, P.; Wang, M.; Li, S.; Morita, H.; Altunbulakli, C.; Reiger, M.; Neumann, A. U.; Lunjani, N.; Traidl-Hoffmann, C.; Nadeau, K. C.; O’Mahony, L.; Akdis, C.; Sokolowska, M., Distribution of ACE2, CD147, CD26, and other SARS-CoV-2 associated molecules in tissues and immune cells in health and in asthma, COPD, obesity, hypertension, and COVID-19 risk factors. Allergy 2020, 75 (11), 2829-2845.

12. Rezaei, M.; Ziai, S. A.; Fakhri, S.; Pouriran, R., ACE2: Its potential role and regulation in severe acute respiratory syndrome and COVID-19. Journal of Cellular Physiology 2020, $n / a(\mathrm{n} / \mathrm{a})$

13. Rehman, S. U.; Shafique, L.; Ihsan, A.; Liu, Q., Evolutionary Trajectory for the Emergence of Novel Coronavirus SARS-CoV-2. Pathogens 2020, 9 (3).

14. Li, Q.; Wu, J.; Nie, J.; Zhang, L.; Hao, H.; Liu, S.; Zhao, C.; Zhang, Q.; Liu, H.; Nie, L.; Qin, H.; Wang, M.; Lu, Q.; Li, X.; Sun, Q.; Liu, J.; Zhang, L.; Li, X.; Huang, W.; Wang, Y., The Impact of Mutations in SARS-CoV-2 Spike on Viral Infectivity and Antigenicity. Cell 2020, 182 (5), 1284-1294.e9.

15. Azzi, L.; Maurino, V.; Baj, A.; Dani, M.; d'Aiuto, A.; Fasano, M.; Lualdi, M.; Sessa, F.; Alberio, T., Diagnostic Salivary Tests for SARS-CoV-2. Journal of Dental Research 2020, 0022034520969670 .

16. Walls, A. C.; Park, Y.-J.; Tortorici, M. A.; Wall, A.; McGuire, A. T.; Veesler, D., Structure, Function, and Antigenicity of the SARS-CoV-2 Spike Glycoprotein. Cell 2020, 181 (2), 281292.e6.

17. Hristov, D.; Pimentel, A. J.; Ujialele, G.; Hamad-Schifferli, K., The immunoprobe aggregation state is central to dipstick immunoassay performance. ACS Applied Materials \& Interfaces 2020, 12 (31), 34620-34629.

18. Tam, J. O.; de Puig, H.; Yen, C.-w.; Bosch, I.; GómezMárquez, J.; Clavet, C.; Hamad-Schifferli, K.; Gehrke, L., A Comparison of Nanoparticle-Antibody Conjugation Strategies in Sandwich Immunoassays. Journal of Immunoassay and Immunochemistry 2017, 38 (4), 355-377.

19. Schneider, C. A.; Rasband, W. S.; Eliceiri, K. W., NIH Image to ImageJ: 25 years of image analysis. Nature Methods 2012, 9 (7), 671-675.

20. Cate, D.; Hsieh, H.; Glukhova, V.; Bishop, J. D.; Hermansky, H. G.; Barrios-Lopez, B.; Grant, B. D.; Anderson, C. E.; Spencer, E.; Kuhn, S.; Gallagher, R.; Rivera, R.; Bennett, C.; Byrnes, S. A.; Connelly, J. T.; Dewan, P. K.; Boyle, D. S.; Weigl, B. H.; Nichols, K. P., Antibody Screening Results for Anti-Nucleocapsid Antibodies Towards the Development of a SARS-CoV-2 Nucleocapsid Protein Antigen Detecting Lateral Flow Assay. ChemRxiv 2020.

21. Rodríguez-Quijada, C.; Gomez-Marquez, J.; HamadSchifferli, K., Repurposing old antibodies for new diseases by exploiting cross reactivity and multicolored nanoparticles. ACS Nano 2020, 14 (6), 6626-6635.

22. Kazakov, V. N.; Udod, A. A.; Zinkovych, I. I.; Fainerman, V. B.; Miller, R., Dynamic surface tension of saliva: General relationships and application in medical diagnostics. Colloids and Surfaces B: Biointerfaces 2009, 74 (2), 457-461.

23. Baliga, S.; Muglikar, S.; Kale, R., Salivary pH: A diagnostic biomarker. Journal of Indian Society of Periodontology 2013, 17 (4), 461-465.

24. Humphrey, S. P.; Williamson, R. T., A review of saliva: Normal composition, flow, and function. Journal of Prosthetic Dentistry 2001, 85 (2), 162-169.

25. Chen, W.-H.; Hotez, P. J.; Bottazzi, M. E., Potential for developing a SARS-CoV receptor-binding domain (RBD) 
recombinant protein as a heterologous human vaccine against coronavirus infectious disease (COVID)-19. Human Vaccines \& Immunotherapeutics 2020, 16 (6), 1239-1242.

26. $\mathrm{Wu}, \mathrm{Y}$., Strong evolutionary convergence of receptorbinding protein spike between COVID-19 and SARS-related coronaviruses. bioRxiv 2020, 2020.03.04.975995.

27. de Puig, H.; Bosch, I.; Carre-Camps, M.; Hamad-Schifferli, $\mathrm{K}$., Effect of the protein corona on antibody-antigen binding in nanoparticle sandwich immunoassays. Bioconjugate Chemistry 2017, $28(1), 230-238$.

28. Cevik, M.; Kuppalli, K.; Kindrachuk, J.; Peiris, M., Virology, transmission, and pathogenesis of SARS-CoV-2. BMJ 2020, 371, m3862.

29. Owusu, M.; Annan, A.; Corman, V. M.; Larbi, R.; Anti, P.; Drexler, J. F.; Agbenyega, O.; Adu-Sarkodie, Y.; Drosten, C., Human Coronaviruses Associated with Upper Respiratory Tract Infections in Three Rural Areas of Ghana. PLOS ONE 2014, 9 (7), e99782.

30. Dijkman, R.; Jebbink, M. F.; Gaunt, E.; Rossen, J. W. A.; Templeton, K. E.; Kuijpers, T. W.; van der Hoek, L., The dominance of human coronavirus OC43 and NL63 infections in infants. Journal of Clinical Virology 2012, 53 (2), 135-139.

31. Heimdal, I.; Moe, N.; Krokstad, S.; Christensen, A.; Skanke, L. H.; Nordbø, S. A.; Døllner, H., Human Coronavirus in Hospitalized Children With Respiratory Tract Infections: A 9-Year Population-Based Study From Norway. The Journal of Infectious Diseases 2019, 219 (8), 1198-1206.

32. Kim, D.; Quinn, J.; Pinsky, B.; Shah, N. H.; Brown, I., Rates of Co-infection Between SARS-CoV-2 and Other Respiratory Pathogens. JAMA 2020, 323 (20), 2085-2086.

33. Kondo, Y.; Miyazaki, S.; Yamashita, R.; Ikeda, T., Coinfection with SARS-CoV-2 and influenza A virus. BMJ Case Reports 2020, 13 (7), e236812.

34. Salvati, A.; Pitek, A. S.; Monopoli, M. P.; Prapainop, K.; Bombelli, F. B.; Hristov, D. R.; Kelly, P. M.; Åberg, C.; Mahon, E.; Dawson, K. A., Transferrin-functionalized nanoparticles lose their targeting capabilities when a biomolecule corona adsorbs on the surface. Nature Nanotechnology 2013, 8 (2), 137-143.

35. Grant, B. D.; Anderson, C. E.; Williford, J. R.; Alonzo, L. F.; Glukhova, V. A.; Boyle, D. S.; Weigl, B. H.; Nichols, K. P., SARS-CoV-2 Coronavirus Nucleocapsid Antigen-Detecting HalfStrip Lateral Flow Assay Toward the Development of Point of Care Tests Using Commercially Available Reagents. Analytical Chemistry 2020, 92 (16), 11305-11309.

36. Wölfel, R.; Corman, V. M.; Guggemos, W.; Seilmaier, M.; Zange, S.; Müller, M. A.; Niemeyer, D.; Jones, T. C.; Vollmar, P.; Rothe, C.; Hoelscher, M.; Bleicker, T.; Brünink, S.; Schneider, J.; Ehmann, R.; Zwirglmaier, K.; Drosten, C.; Wendtner, C., Virological assessment of hospitalized patients with COVID-2019. Nature 2020, 581 (7809), 465-469.

37. Bar-On, Y. M.; Flamholz, A.; Phillips, R.; Milo, R., SARSCoV-2 (COVID-19) by the numbers. Elife 2020, 9, e57309.

38. Wang, Y.; Zhang, L.; Sang, L.; Ye, F.; Ruan, S.; Zhong, B.; Song, T.; Alshukairi, A. N.; Chen, R.; Zhang, Z.; Gan, M.; Zhu, A.; Huang, Y.; Luo, L.; Mok, C. K. P.; Al Gethamy, M. M.; Tan, H.; Li, Z.; Huang, X.; Li, F.; Sun, J.; Zhang, Y.; Wen, L.; Li, Y.; Chen, Z.; Zhuang, Z.; Zhuo, J.; Chen, C.; Kuang, L.; Wang, J.; Lv, H.; Jiang, Y.; Li, M.; Lin, Y.; Deng, Y.; Tang, L.; Liang, J.; Huang, J.; Perlman, S.; Zhong, N.; Zhao, J.; Malik Peiris, J. S.; Li, Y.; Zhao, J., Kinetics of viral load and antibody response in relation to COVID-19 severity. The Journal of Clinical Investigation 2020.

39. Pan, Y.; Zhang, D.; Yang, P.; Poon, L. L. M.; Wang, Q., Viral load of SARS-CoV-2 in clinical samples. The Lancet Infectious Diseases 2020, 20 (4), 411-412.

40. Weissleder, R.; Lee, H.; Ko, J.; Pittet, M. J., COVID-19 diagnostics in context. Science Translational Medicine 2020, 12 (546), eabc1931.

41. Russo, L.; Sanchez-Purra, M.; Rodríguez-Quijada, C.; Leonardo, B. M.; Puntes, V.; Hamad-Schifferli, K., Detection of resistance protein A (MxA) in paper-based immunoassays with surface enhanced Raman spectroscopy with $\mathrm{AuAg}$ nanoshells. Nanoscale 2019, 11 (22), 10819-10827
42. Sánchez-Purrà, M.; Carré Camps, M.; de Puig Guixé, H.; Bosch, I.; Gehrke, L.; Hamad-Schifferli, K., SERS-based sandwich immunoassays for multiplexed detection of Zika and dengue viral biomarkers. ACS Infectious Diseases 2017, 3 (10), 767-776.

43. Babak Y. Moghadam, K. T. C., and Jonathan D. Posner, Two Orders of Magnitude Improvement in Detection Limit of Lateral Flow Assays Using Isotachophoresis. Analytical Chemistry 2015, 87, 1009-1017.

44. Qin, Z.; Chan, W. C. W.; Boulware, D. R.; Akkin, T.; Butler, E. K.; Bischof, J. C., Significantly Improved Analytical Sensitivity of Lateral Flow Immunoassays by Using Thermal Contrast. Angewandte Chemie International Edition 2012, 51 (18), 4358-4361.

45. Vashist, S. K.; Luppa, P. B.; Yeo, L. Y.; Ozcan, A.; Luong, J. H. T., Emerging Technologies for Next-Generation Point-of-Care Testing. Trends in Biotechnology 2015, 33 (11), 692-705.

\section{SYNOPSIS TOC}

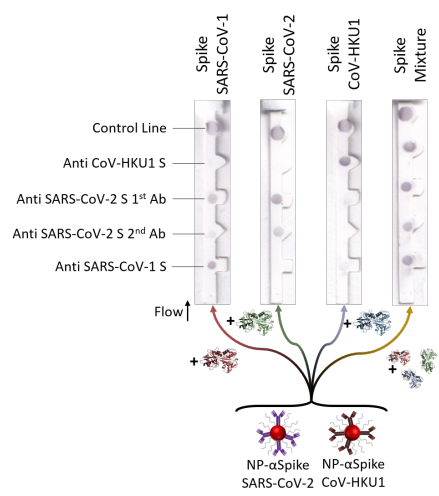

